\title{
Análisis de diferentes fechas de establecimiento de Santiago de Veraguas. Identificación de posible fecha fundacional.
}

\author{
Sebastián Ariel Aguilar Medina ${ }^{1 *}$ \\ ${ }^{1}$ Escuela de Arquitectura, Centro Regional de Veraguas, Universidad Católica Santa María La \\ Antigua (USMA).
}

*Autor para correspondencia. Email: arq.sebastianaguilar@yahoo.com

Recibido: 3 de junio de 2019

Aceptado: 15 de agosto de 2019

\begin{abstract}
Resumen
Existen diferentes teorías sobre la fundación de la ciudad de Santiago de Veraguas, pero aún no se sabe con certeza la fecha real de su fundación, después de arduos esfuerzos de historiadores y otros investigadores sociales, que han buscado la respuesta a esta pregunta y que están muy distantes entre sí en términos de fechas. Algunos coinciden con momentos específicos que describiremos en este artículo. Esta evaluación se ha centrado en varias teorías sobre posibles fechas de fundación de la ciudad, de acuerdo a documentos estudiados de la época y la investigación contemporánea de profesionales en el campo. Se presenta un trabajo teórico, que integra la metodología de investigación de información historiográfica, con el objetivo de motivar el desarrollo de la investigación relacionada con el tema de manera científica y obtener resultados que puedan ser utilizados como material con una base didáctica y técnica en las futuras investigaciones de profesionales, estudiantes y para el uso cultural de toda la población panameña e internacionalmente también.

Palabras o frases claves: Santiago, Veraguas, Panamá, Historia colonial, Patrimonio Arquitectónico, Fecha fundacional.
\end{abstract}

\begin{abstract}
There are different theories about the founding of the city of Santiago de Veraguas but it is not yet known for sure, the real date of its foundation, after arduous efforts of historians and other social researchers, who have sought the answer to this question, which are very distant from each other and some coincide with specific dates that we will describe in this article. This evaluation has focused on several theories of possible dates of foundation of the city, according to documents studied of the time and contemporary research of professionals in the field. A theoretical work is presented, which integrates the methodology of research of historiographic information, aimed to motivate the development of the research related to the subject in a scientific way and to obtain results that can be used as material with a didactic and technical basis in future investigations of professionals, students and for the cultural use of the entire Panamanian population and internationally as well.
\end{abstract}

Key Words: Santiago, Veraguas, Panamá, Colonial History, Architectural heritage, Foundational date. 


\section{Introducción.}

Uno de los grandes dilemas en la historia de la ciudad de Santiago de Veraguas, es desconocimiento de una fecha comprobada de fundación para la ciudad, a pesar de grandes esfuerzos realizados por diferentes investigadores e historiadores por varios años, no se llega al consenso de una fecha específica para dicho evento de la historia. Según la Real Academia Española de la Lengua (RAE), define fundación como, del latin Fundationis, principio, erección, establecimiento y origen de algo. Documento en que constan las cláusulas de una institución de mayorazgo, de una obra pía, etc.

Iniciamos la investigación desde la fecha probable de fundación de Santiago de Veraguas, aunque aún no se sabe a ciencia cierta, la fecha real de la fundación de esta importante ciudad panameña, se manejan varias teorías y varias fechas, unas muy distantes de otras.

Dentro de las fechas que diferentes historiadores han propuesto les dejamos las siguientes:

\subsection{Santiago de Veragua en 1518. Siglo XVI.}

Algunos autores establecen la fundación de la ciudad de Santiago de Veragua, hacia el año 1518 fundada por Diego de Alvítez y Gaspar de Espinoza (basados en cfc. Antonio CuervoDocumentos inéditos. Vol. 11, pág. 342), esta fundación fue en las grandes sabanas a orillas del Rio Martin Grande cerca del balneario "Los Chorros" área que luego fue abandonada por los constantes ataques de las tribus indígenas. Aunque en otros documentos, se menciona que el capitán Diego de Albitez recibe permiso de su jefe Pedrarias para colonizar Veragua, pero nunca llegará allí, e intenta poblar este año Portobelo (Panamá).

Dentro de los autores que coinciden con esta fecha (1518), se encuentra el Reverendo Vidal Fernández de Palomares (1904 -1997), el cual la menciona en un artículo publicado el 29 de Noviembre de 1,953 en el diario "El Lábaro", también los Hermanos Antonio de Ulloa y Jorge Juan de Ulloa "Viaje a la America Meridional" 1736, los relatos tradicionales de Don Luis E. de Fábrega originario de Santiago, Berthold Seeman Historia General de Panamá Siglo XIX) (Botello Pino, E. 2009)

\subsection{Santiago de Veragua (doctrinera) 1560. Siglo XVI.}

Esta fecha es mencionada por el historiador Rubén Darío Carles, en la definición de Santiago como Distrito de Veragua (Botello Pino, E. 2009)

\subsection{Santiago de Veragua (doctrinera) 1573. Siglo XVI.}

Hay otra teoría de la Investigadora Esilda Isabel Botello Pino, que, basándose en los argumentos del historiador coclesano Gaspar Rosas Quirós, quien desarrolla la temática de las doctrinas y parroquias encomendaderas en el área de Veraguas, la lleva a proponer, la primera instancia del pueblo como una doctrina o asiento de indios para doctrina o encomienda en las cercanías del Río Los Chorros. Ella sustenta que la primera fase fue la doctrina luego parroquia doctrinera y subirla luego a ciudad, y la fecha que surge Santiago como encomienda doctrinera seria en 1573, también para esta misma fecha, mencionada en el libro "Conquista, Evangelización y Resistencia" de Alfredo Castillero Calvo, donde denomina “Los Llanos de Santiago" (Botello Pino, E. 2009). 


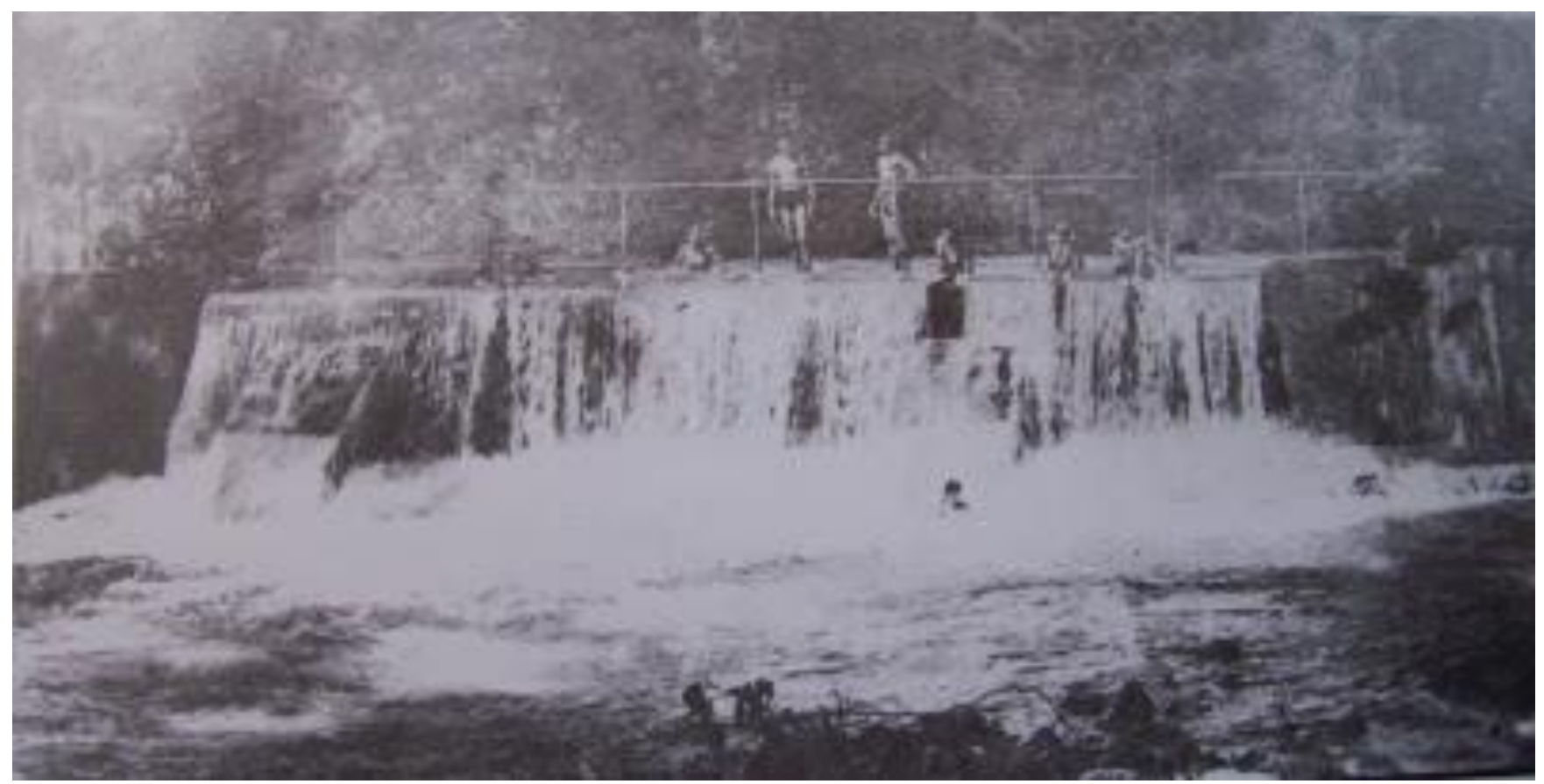

Ilustración 1. Foto del Rio los Chorros. Antiguo Balneario.

1.4 Teoría de 1614:

El escritor Veragüense Nicolás Caballero, en su libro "Síntesis Histórica de Santiago de Veraguas" propone dos fechas la de 1614 de la primera ciudad y la de 1636 como traslado casi coincidiendo con Mario Molina en esta última (Caballero, N. 1998). La investigadora Esilda Botello Pino indica esta fecha como la denominación de Santiago en parroquia Doctrinera en 1614, coincidiendo con la fecha que describe Nicolás Caballero (Botello Pino, E. 2009).

\subsection{Santiago de Veragua 1620-1621. (Santiago de Veragua la Vieja),}

En esta fecha coinciden muchos autores, dentro de ellos el cronista Juan Diez De La Calle en su obra "Colección de Documentos de la Historia Eclesiástica y Civil de América", que indica que, debido a motivos de catequización y el agotamiento aurífero del norte de Veragua las autoridades ordenaron a ambos grupos encontrarse en un punto en el centro de la región paras ser poblado por habitantes de San Pedro de Montijo y de Santa Fe, los cuales se encontraron en el Sitio que hoy se conoce como Santiago de Veraguas, realizando su fundación en 1621 el 23 de Octubre, se establece como fecha de fundación de la ciudad de Santiago de Veragua (Santiago de Veragua la Vieja), y según indagaciones del historiador Osorio Osorio en la "Biblioteca Nacional de Madrid" en el Manuscrito 2930, describe que ya para esta fecha 1621, Santiago de Veragua La Vieja, como luego fue denominada ya existía. (Folio 159).

Otros autores establecen 1620, como Rubén Darío Carles Oberto (1896-1981) y Samuel Kirkland Lothrop (1892-1965) (Botello Pino, E. 2009). 
Esilda Botello Pino, coincide con Mario Molina en la fundación de la ciudad y Villa la llamada por Molina "Santiago la Vieja" como fecha probable 162001621.

Algunos tratan de visualizar la relación con la fundación de San Francisco de la Montaña (1621), colocando a Santiago como posible Parroquia Doctrinera, como Castillero Calvo y Rubén Darío Carles (Botello Pino, E. 2009).
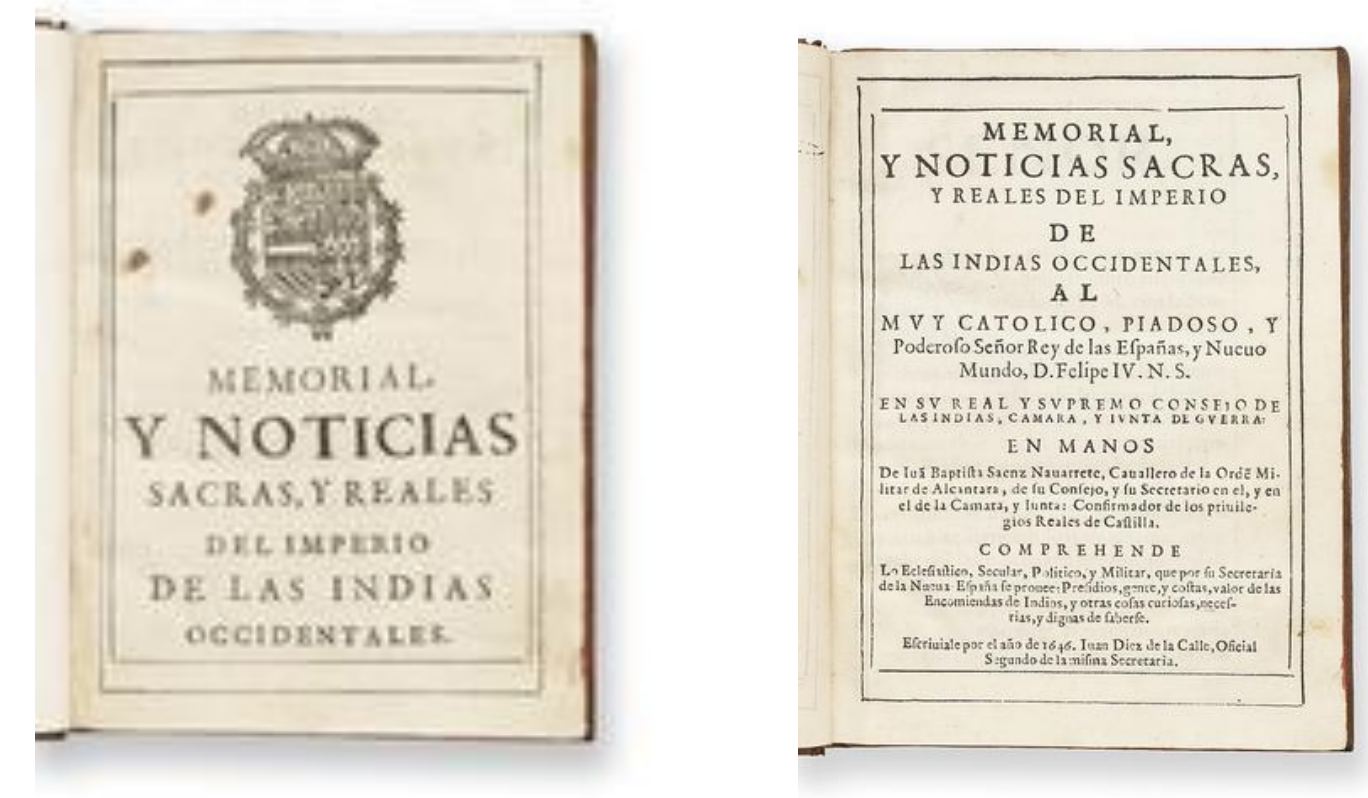

Ilustración 2. "Colección de Documentos de la Historia Eclesiástica y Civil de América", Juan Diez De La Calle.

\subsection{Santiago de Veragua 1635-1636-1637-1638. (Santiago de Veragua la nueva).}

En la obra "Coclé de Natá" del investigador Gaspar Rosas Quiroz, comenta la fecha de 1635. Pero Según el Dr. Mario Molina, entre 1620 y 1636 existe una transición importante en el esquema poblacional de Santiago de Veragua, se construye un nuevo templo, un hospital, viviendas y trazado de las calles y plaza (Molina, M. 2013).

La tercera fecha importante en la evolución Colonial de Santiago de Veraguas es 1637, en esta fecha Santiago de Veragua La Vieja experimenta cambios de sitio, se Reconstruye E1 Templo, se realiza la primera construcción de un Hospital en la ciudad (Hospital San Juan de Dios), el principal gestor de estas obras es el Lic. Martin Delgado y Llanos cura vicario. Y se realiza el trazado de las principales calles y plazas. (Molina, M. 2013)

En sus inicios solo se contaba con dos calles, La Calle Real, hoy Calles segunda que se y la Calle del Calvario, hoy Avenida Central, en sus inicios hasta la actual Biblioteca Publica lugar donde se encontraba el Hospital y asilo San Juan de Dios y la Iglesia (Molina, M. 2013).

Según el escritor Nicolás de J. Caballero, en su libro "Síntesis Histórica de Santiago de Veraguas", establece la fundación de Santiago en 1636 y como fundador a Enrique Enríquez 
presidente de la Audiencia el cual comunica al Rey que los dos poblados de la Gobernación de Veragua, serán reducidos en un solo poblado, los pueblos llamados Santa Fe y Montijo, lo que indica que Santiago fue fundado en 1636 (Caballero, N. 1998). El historiador Alfredo Castillero Calvo, coincide también con esta fecha.

En lo que podemos denominar su tercera y última fundación en 1637 se fusionan los pobladores de las comunidades de Santa Fe y San Pedro de Montijo ciudades que fueron abandonadas después de los problemas en las actividades de las minas de oro.

Santiago de Veragua al ser trasladada a su nueva ubicación en 1637, según Molina, se convirtió en un centro operacional de abastecimiento de su vecindario y de los pueblos aledaños cercanos, sobre todo de los pueblos mineros con La Concepción (en el actual distrito de Santa Fe) y luego Aguacatal y Libertad (en el actual distrito de San Francisco de la Montaña) (Molina, M. 2013).

En 1638, se hacen muchas reconstrucciones a las estructuras existentes y se crean nuevas edificaciones y calles que van a cambiar para siempre la fisionomía de la ciudad de Santiago de Veragua (Caballero, N. 1998).

La investigadora, Esilda Botello Pino, coincide con Mario Molina en el traslado de la ciudad Villa actual (trasladada a la posición actual) entre 1636-1637 (Botello Pino, E. 2009).

Como verán en los escritos presentados en esta investigación, se habla del traslado de la ciudad en 1636 y las edificaciones y trazado de calles que luego se realizaron a partir de esa fecha en el lugar donde hoy está ubicada la ciudad de Santiago de Veragua.

Ya para 1640, hay constancia de la existencia de la ciudad por medio de la Relación de Juan Requejo Salcedo de 1640, que, en la lista de ciudades de Veragua, cita ya la ciudad de "Santiago de Veragua" (Molina, M. 2013).

Para 1650 la ciudad inicia su papel preponderante al ser incorporada, Santiago de Veragua, a la ruta urbanística del Camino Real de Recuas de Mulas procedente de Centroamérica rumbo a la ciudad de Panamá, esta ruta se habilitó completamente a partir de 1650 (Molina, M. 2013).

También en 1659 Diez de la Calle menciona en las poblaciones españolas de Veragua a "Santiago de Veragua".

En 1661, el Obispo de Panamá, que visitaba Veragua precisa la ubicación de Veragua, detallando que "en la Gobernación de Veragua, están agregados dos pueblitos indios el de San Francisco y Atalaya, al mando de españoles de Santiago a una distancia de 2 leguas (unos $10 \boldsymbol{u} \mathbf{1 1} \mathbf{~ k m}$ ), es la distancia que está actualmente Atalaya de Santiago (Molina, M. 2013).

\section{Metodología}

La presente investigación se fundamentó mediante el trabajo de campo, la revisión de fuentes bibliográficas y documentación en archivos. Se emplearon métodos teóricos: histórico-lógico, sistémico-estructural y análisis-síntesis; y el método empírico: observación de la realidad.

La metodología y las variables de estudio, se definieron para establecer una probable fecha de Fundación de Santiago de Veraguas, objetivo principal de la investigación. Partió de un análisis integral 
que contempló el marco histórico en que surge y se desarrolla la historia y urbanismo de la ciudad de Santiago de Veraguas, en el contexto nacional y local, el estudio de los referentes historiográficos relacionados con el tema, los aspectos de carácter teórico-metodológico, los criterios sobre estudios tipológicos y las aportaciones del medio geográfico en que se enclava el objeto de estudio.

En la bibliografía consultada, hemos encontrado, algunos temas como la definición del período general de desarrollo, así como etapas evolutivas vinculadas a los factores de orden socioeconómico y cultural en cada contexto, diversidad de temas arquitectónicos (Fabian Garré, 2001), (Puente San Millán, 2014). Asimilación de códigos por las distintas clases sociales, relacionados con las tipologías constructivas llevadas a cabo, tanto por profesionales como por constructores y usuarios.

Se Utilizaron métodos tradicionales como de vanguardia tales como: la incorporación del uso de aplicaciones informáticas y herramientas en línea que permitieron automatizar procesos tediosos de búsqueda y gestión bibliográfica; la revisión crítica, de artículos de investigación y lecturas existentes; la revisión de estadísticas existentes y aplicándolas al problema en investigación; la utilización de métodos gráficos para resultados de los datos; la revisión de aspectos metodológicos de proyectos ya realizados dentro del área o en otras ramas; la revisión de publicaciones donde suelen difundirse los resultados de investigación y la producción académica o artística de los profesionales del área.

Se realizaron el análisis de las diferentes teorías, por ejemplo, Molina Castillo, M. J. (2013), Castillero Calvo, A. (1967), (Botello Pino, E. 1995), Reverendo Vidal Fernández de Palomares (1904 -1997), Hermanos Antonio de Ulloa y Jorge Juan de Ulloa (1736), Berthold Seeman Historia General de Panamá Siglo XIX), Jaén Suarez, O. (1999), Caballero, N (1960), Carles, R.D. (1969), Osorio Osorio, A (2000)

Todas estas fuentes (libros, revistas científicas, periódicos, informes técnicos y de investigación de instituciones públicas y normas técnicas.) fueron dirigidas directamente a extraer información sobre la evolución, fundación, crecimiento y características de la ciudad de Santiago de Veraguas, limitándonos en las áreas de estudio del urbanismo y la arquitectura. Utilizamos un patrón de análisis para los datos cuantitativos o cualitativos que demandó la integración de aspectos que provienen, en parte, de la extensa literatura disponible en el ámbito de la investigación y la estadística (Grout, 2010), (Francesc Caballé I Esteve, 2003). Otra parte proviene de la experiencia que acumulamos en el campo de trabajo y la frecuencia con que realiza este tipo de labor.

\section{Resultados}

Luego de la aplicación de los diferentes métodos de análisis ya mencionados, presentamos el cuadro depurado que resume las teorías que poseen suficiente sustento historiográfico.

\section{Cuadro 1. Teorías sobre fundación de la Ciudad de Santiago de Veraguas.}

\begin{tabular}{|l|l|l|l|l|}
\hline Año & Teoría & documento & lugar & Citada \\
\hline 1518 & $\begin{array}{l}\text { Fundada por } \\
\text { Diego de Alvítez } \\
\text { y Gaspar de } \\
\text { Espinoza }\end{array}$ & $\begin{array}{l}\text { Basados en cfc. } \\
\text { Antonio Cuervo- }\end{array}$ & $\begin{array}{l}\text { En las } \\
\text { grandes } \\
\text { sabanas a } \\
\text { orillas del }\end{array}$ & $\begin{array}{l}\text { - (Botello Pino, Esilda. 1995) } \\
\text {-Reverendo Vidal Fernández de } \\
\text { Palomares (1904 -1997) }\end{array}$ \\
\hline
\end{tabular}




\begin{tabular}{|c|c|c|c|c|}
\hline & & $\begin{array}{l}\text { inéditos. Vol. 11, } \\
\text { pág. } 342\end{array}$ & $\begin{array}{l}\text { Rio Martin } \\
\text { Grande } \\
\text { cerca del } \\
\text { balneario } \\
\text { "Los } \\
\text { Chorros" }\end{array}$ & $\begin{array}{l}\text {-Hermanos Antonio de Ulloa y } \\
\text { Jorge Juan de Ulloa } 1736 . \\
\text {-relatos tradicionales de Don } \\
\text { Luis E. de Fábrega. } \\
\text {-Berthold Seeman Historia } \\
\text { General de Panamá Siglo XIX). }\end{array}$ \\
\hline 1560 & $\begin{array}{l}\text { Santiago } \\
\text { Doctrinera }\end{array}$ & $\begin{array}{l}\text { mencionada por } \\
\text { el historiador } \\
\text { Rubén Darío } \\
\text { Carles }\end{array}$ & & (Botello Pino, Esilda. 1995) \\
\hline 1573 & $\begin{array}{l}\text { Santiago como } \\
\text { encomienda } \\
\text { doctrinera. } \\
\text { "Los Llanos de } \\
\text { Santiago" }\end{array}$ & $\begin{array}{l}\text { "Conquista, } \\
\text { Evangelización y } \\
\text { Resistencia" de } \\
\text { Alfredo Castillero } \\
\text { Calvo. }\end{array}$ & $\begin{array}{l}\text { las cercanías } \\
\text { del Río Los } \\
\text { Chorros }\end{array}$ & $\begin{array}{l}\text { Gaspar Rosas Quirós. } \\
\text { (Botello Pino, Esilda. 1995) }\end{array}$ \\
\hline 1607 & $\begin{array}{l}\text { "Jurisdicción de } \\
\text { Santiago" }\end{array}$ & $\begin{array}{l}\text { El Archivo } \\
\text { General de } \\
\text { Indias, en } 1607\end{array}$ & & $\begin{array}{l}\text { del Dr. Omar Jaén Suarez. } \\
\text { (1999) }\end{array}$ \\
\hline 1607 & $\begin{array}{l}\text { "Elpueblo del Rio } \\
\text { Martín" }\end{array}$ & $\begin{array}{l}\text { informe de Obispo } \\
\text { Antonio Calderón }\end{array}$ & $\begin{array}{l}\text { "Elpueblo del } \\
\text { Rio Martín" }\end{array}$ & $\begin{array}{l}\text { (Molina Castillo, Mario José. } \\
\text { 2013) }\end{array}$ \\
\hline 1609 & $\begin{array}{l}\text { Legalidad de la } \\
\text { creación de } \\
\text { Santiago de } \\
\text { Veragua. }\end{array}$ & $\begin{array}{l}\text { Cedula fechada } \\
\text { en Madrid el } 19 \\
\text { de enero de } 1609 \\
\text { La Corona } \\
\text { aceptaba la } \\
\text { solicitud de la } \\
\text { Audiencia. la } \\
\text { unificación de } \\
\text { Montijo y Santa } \\
\text { Fe. }\end{array}$ & & $\begin{array}{l}\text { (Molina Castillo, Mario José. } \\
\text { 2013) }\end{array}$ \\
\hline 1614 & $\begin{array}{l}\text { Primera ciudad. } \\
\text { parroquia } \\
\text { Doctrinera }\end{array}$ & $\begin{array}{l}\text { "Síntesis } \\
\text { Histórica de } \\
\text { Santiago de } \\
\text { Veraguas" } \\
\text { Informe Esilda } \\
\text { Botello Pino }\end{array}$ & & $\begin{array}{l}\text { Nicolás Caballero } \\
\text { Esilda Botello Pino }\end{array}$ \\
\hline
\end{tabular}

\section{Discusión y Conclusiones.}

En la bibliografía consultada, hemos encontrado algunos temas como la definición del período general de desarrollo, así como las teorías de posibles fechas de fundación realizadas por expertos en el tema.

Toda disciplina o profesión necesita una base documentada para realizar propuestas, investigaciones y diagnósticos, efectivos para soluciones a un sinnúmero de problemas que hoy afectan nuestras ciudades. Sin tomar en cuenta los antecedentes y la evolución de las mismas es muy difícil llegar a resultados satisfactorios de cualquier tipo que se realicen en un área específica. 
En este artículo, se plasma de manera profunda y científica dicha documentación, que es necesaria en la ciudad de Santiago, poca estudiada desde el punto de vista histórico, para comprender sus inicios hasta la fecha.

Afloran de esta investigación, el análisis de la problemática de no contar con una fecha de fundación determinada, la cual puede causar otras consecuencias, como el desconocimiento de los valores históricos y culturales de parte de los pobladores locales y visitantes; la persistencia de prejuicios hacia el patrimonio histórico, irrespetando la identidad local y regional; la limitada tutela y manejo del patrimonio histórico por parte de los organismos del territorio, por falta de información científica necesaria.

Tomando en cuenta los aspectos planteados con anterioridad, la situación problemática consiste en la necesidad de profundizar en los estudios acerca del patrimonio histórico en la ciudad de Santiago de Veraguas, para completar los vacíos existentes en temas culturales específicos como la fundación de esta ciudad.

La confusión en identificar la fecha de fundación tiene varias causas, una debido a que no se han encontrada acta fundacional, sino escritos de referencia indirecta y la otra es por la falta de concordancia de la categoría del pueblo a través de las fechas, su evolución de categoría poblacional, posiblemente de Encomienda a parroquia, de parroquia a ciudad (Botello Pino, E. 2009).

Lo cierto que a la fecha no se ha llegado a una concordancia entre historiadores e investigadores en cuanto a la fecha real de fundación de la ciudad de Santiago de Veraguas, la cual ha sido muy estudiada y de la cual nosotros brindamos, con el análisis de las variables existentes, nuestras conclusiones sobre el tema.

De aquí definimos que el principal problema para determinar la fecha de la fundación de Santiago de Veraguas es el empleo de las categorías de emplazamientos existentes en área.

Primero debemos tomar en cuenta el concepto de Ciudad, el cual para el Siglo XVI y XVII, época de visita de los primeros españoles a nuestras tierras era categorizado por los siguientes elementos, para que a un poblado se le diera Título de Ciudad debía contar dos características, Orden Administrativa y el linaje de sus pobladores (en este caso de Linaje puro español). Santiago cuenta con estas características solo a partir del Siglo XVII, cuando se impone la cedula real fechada en Madrid el 19 de enero de 1609, donde La Corona aceptaba la solicitud de la Audiencia y ordenaba la unificación de Montijo y Santa Fe. Esta Cédula nace del informe de 1608, cuando la Audiencia informa a La Corona que sería conveniente refundir la población de Santa Fe con los pobladores de Montijo, en un lugar más salubre. De esta manera queda definitivamente la legalidad de la creación de Santiago de Veragua. El único detalle que no está claro es cuando exactamente se aplicó con fecha exacta, la fundación del poblado, por lo cual presentamos nuestra posición sobre este tema, en la cual, aceptamos como fecha más probable de la fundación de la ciudad de Santiago, basándonos en los criterios de jerarquización y categorización Colonial sobre la Titulación de ciudades, la fecha del 23 de octubre de 1621, (con documento de la "Biblioteca Nacional de Madrid" en el Manuscrito 2930, Folio 159), también tomando en cuenta la coincidencia en la fecha de fundación de otros poblados para la misma fecha como San Francisco de la Montaña (1621) y otro documento de apoyo de esta fecha es el del cronista Juan Diez De La Calle en su obra "Colección de Documentos de la Historia Eclesiástica y Civil de América”, donde establece esta fecha como fundación de la ciudad, 
lo cual recalcamos, que existen otras evidencias de que ya existían pobladores en el área para esta época, pero mas no así la categorización para ubicar el título de ciudad a Santiago de Veraguas.

Como referencia, se documenta, la puesta en práctica de este plan queda a cargo del Oidor Fiscal Bartolomé de Morquecho quien visita Montijo en 1611 donde fundara San Bartolomé de Tabarabá, lo cual nos indica que antes de 1611 no se realiza la fundación por lo cual cualquier otra teoría sobre fechas anteriores a esta la descartamos.

La evidencia de los primeros residentes españoles en Santiago tiene como antecedentes los poblados de Montijo y Santa Fe, los cuales según Castillero Calvo y el Dr. Molina, inician con la despoblación de Santa Fe a fines del Siglo XVI (1589) y principio del Siglo XVII (1600), cuando los pocos vecinos que quedaron en esta población marcharon hacia las sabanas a ciertas estancias y haciendas que quedaban en el Sitio El Naranjal, a orillas del rio Escoria o rio Santa María. En 1604 con la visita pastoral del Obispo Antonio Calderón, los santafereños muestran su deseo de que Santa Fe fuera trasladada a El Sitio E1 Naranjal, donde se encontraban la mayoría de los vecinos en esa época. Para 1607 existen pocos poblados y pobladores en Veraguas y Francisco Valverde y Mercado menciona a Montijo como el poblado más cercano de Santa Fe, por lo cual se deduce que para 1607 no existe aún el poblado de Santiago de Veragua, como ciudad.

Aquí definimos las jerarquías de asentamientos estudiadas, utilizadas para la sustentación y descarte de la categoría de ciudad. Aquí se menciona las siguientes denominaciones:

1. Santiago Doctrinera, (tomado de Centro Doctrinero). Edificados en zonas de alta densidad indígena y con el objetivo de convertir a los indios a la fe católica. Un centro doctrinero completo constaba de una estructura espacial (una "plaza"), y cinco elementos básicos aunque no siempre aparecen todos-, que son: un templo (de una sola nave, para españoles), una capilla abierta (o cobertizo, para indios), una casa cural, unas capillas posas (para "sentar" los santos en las procesiones) y una cruz atrial (representación simbólica del nuevo credo, puesta en el centro de la plaza). (Valdeón, Julio; Salrach, José María; Zabalo, Javier (1987).)

La importancia del centro doctrinero no radica en su excelencia arquitectónica, de hecho, eran construcciones muy sencillas, sino en su condición de representar un sistema de ordenamiento espacial urbano alternativo a la fundación formal de ciudades. De las pocas muestras subsistentes, el más completo es el centro doctrinero de Sutatausa, que posee el templo original y las cuatro capillas posas ( 3 originales, 1 reconstruida).

De aquí también desprendemos, la desaparición del primer asentamiento Doctrinal que el Doctor Molina denominó, Santiago La Vieja, la cual proponemos que se fundó en 1621.

2. Santiago de Encomienda Doctrinera (Encomienda Colonial): La encomienda fue una institución implementada por los conquistadores españoles durante la colonización en América, para sacar provecho del trabajo indígena. Consistía en la entrega de un grupo de indios a un español para que éste los protegiera, educara y evangelizara. Aquellos debían pagar un tributo como obligación de "vasallos" de la Corona, retribuyendo de esta manera los servicios prestados por el encomendero. Generalmente, este tributo se pagaba con trabajo, 
pero existieron diferencias regionales. (Valdeón, Julio; Salrach, José María; Zabalo, Javier (1987).)

Esta descrita en la teoría de 1573, identifica que existe ya presencia española pero no como ciudad sino como encomienda.

3. Jurisdicción de Santiago (Jurisdicción Señorial o Señorío): El señorío es una institución propia de la Edad Media y la Edad Moderna en España, en cierto modo similar al feudo del Imperio carolingio. Surgió en los reinos cristianos del norte peninsular y se extendió con la Reconquista al resto del territorio, confirmándose e incrementándose (refeudalización) con la Monarquía Hispánica posterior. (Valdeón, Julio; Salrach, José María; Zabalo, Javier (1987).)

Se trata de una donación hereditaria de tierras y vasallos, incluida la jurisdicción, dada por monarcas a nobles o clérigos como pago por servicios prestados o recompensa a méritos adquiridos, pero por su mera voluntad (merced).

De esta forma vemos que la denominación es más administrativa que en función de la fisionomía de una ciudad propiamente dicha, por lo cual no es consecuente directamente con la fundación de la ciudad de Santiago de Veraguas, más si le da carácter administrativo a la misma la cual está por formalizarse luego con la Cedula Real de 1609.

En el siguiente cuadro valoramos las teorías antes propuestas para la fundación de la ciudad de Santiago de Veraguas, en el cual, basándonos en la categorización de ciudad como Titulo Colonial, realizamos la aceptación o descartamos la misma, tomando en cuenta lo antes descrito.

Cuadro 2. Cuadro comparativo-analítico sobre Teorías sobre fundación de la Ciudad de Santiago de Veraguas.

\begin{tabular}{|l|l|l|}
\hline Año & Teoría & \multicolumn{1}{c|}{ Aceptada o descartada } \\
\hline $\mathbf{1 5 1 8}$ & $\begin{array}{l}\text { Fundada por } \\
\text { Diego de Alvítez } \\
\text { y Gaspar de } \\
\text { Espinoza }\end{array}$ & $\begin{array}{l}\text { Descartada como ciudad. } \\
\text { Podemos categorizarla como asentamiento de reconocimiento o de } \\
\text { campaña. Posible ocupación de pobladores autóctonos. }\end{array}$ \\
\hline $\mathbf{1 5 6 0}$ & $\begin{array}{l}\text { Santiago } \\
\text { Doctrinera }\end{array}$ & $\begin{array}{l}\text { Descartada como ciudad. } \\
\text { Es claramente categorizada como Centro Doctrinero, de población } \\
\text { indígena en su mayoría. }\end{array}$ \\
\hline $\mathbf{1 5 7 3}$ & $\begin{array}{l}\text { Santiago como } \\
\text { encomienda } \\
\text { doctrinera. } \\
\text { "Los Llanos de } \\
\text { Santiago" }\end{array}$ & $\begin{array}{l}\text { Descartada como ciudad. } \\
\text { Es claramente categorizada como Encomienda Doctrinera, de } \\
\text { población indígena en su mayoría. }\end{array}$ \\
\hline $\mathbf{1 6 0 7}$ & $\begin{array}{l}\text { "Jurisdicción de } \\
\text { Santiago" }\end{array}$ & Descartada como ciudad. \\
\hline
\end{tabular}




\begin{tabular}{|c|c|c|}
\hline & & $\begin{array}{l}\text { Es claramente categorizada como jurisdicción lo cual es un término } \\
\text { más administrativo que de estructura de ciudad. }\end{array}$ \\
\hline 1607 & $\begin{array}{l}\text { "Elpueblo del Río } \\
\text { Martín" }\end{array}$ & $\begin{array}{l}\text { Descartada como ciudad. } \\
\text { Es la mención del mismo Centro Doctrinero, de población indígena } \\
\text { en su mayoría. }\end{array}$ \\
\hline 1609 & $\begin{array}{l}\text { Legalidad de la } \\
\text { creación de } \\
\text { Santiago de } \\
\text { Veragua. }\end{array}$ & $\begin{array}{l}\text { Fecha cuando se proclama legalmente la creación de la ciudad, } \\
\text { la cual pasarán varios años para que se establezca físicamente. }\end{array}$ \\
\hline 1614 & $\begin{array}{l}\text { Primera ciudad. } \\
\text { parroquia } \\
\text { Doctrinera }\end{array}$ & $\begin{array}{l}\text { Descartada como ciudad. } \\
\text { Es la mención del mismo Centro Doctrinero, de población indígena } \\
\text { en su mayoría }\end{array}$ \\
\hline $\begin{array}{l}1620- \\
1621\end{array}$ & $\begin{array}{l}\text { Parroquia } \\
\text { Doctrinera. } \\
\text { Fundación en } \\
1621 \text { el } 23 \text { de } \\
\text { octubre. } \\
\text { (Santiago de } \\
\text { Veragua la } \\
\text { Vieja), }\end{array}$ & $\begin{array}{l}\text { Aceptada como ciudad. } \\
\text { Es la primera vez cuando ya se integran a la ciudad los pobladores de } \\
\text { linaje español provenientes de los poblados de Montijo y Santa Fe y } \\
\text { con su Orden Administrativo. }\end{array}$ \\
\hline 1635 & & \multirow{2}{*}{$\begin{array}{l}\text { A partir de esta fecha se inicia el traslado a la actual ubicación de la } \\
\text { ciudad, denominada Santiago La Nueva y su posterior crecimiento. }\end{array}$} \\
\hline 1636 & $\begin{array}{l}\text { Traslado de la } \\
\text { ciudad (Santiago } \\
\text { La Nueva) }\end{array}$ & \\
\hline
\end{tabular}

En resumen, el presente estudio confirma que, con base a diversas evidencias, la fecha más plausible de fundación de la Ciudad de Santiago de Veraguas es, efectivamente, el 23 de octubre de 1621.

\section{Bibliografía}

- Valdeón, Julio; Salrach, José María; Zabalo, Javier (1987). Feudalismo y consolidación de los pueblos hispánicos. Barcelona Labor. ISBN 84-335-9424-9.

- Molina Castillo, M. J. (2013). Veragua: Tierra de Colón y de Urraca. Panamá, Arte Gráfico Impresores.

- Puente San Millan (2014). Arquitectura Art Decó en el Centro Histórico de la ciudad de Santiago de Cuba. Editorial: Facultad de Arquitectura. Universidad Tecnológica de La Habana José Antonio Echeverría, Cujae. La Habana. Cuba.

- Garré, F. (2001.) Patrimonio arquitectónico urbano, preservación y rescate: bases conceptuales e instrumentos de salvaguarda. Revista Conserva 2001. Argentina.

- Caballé I Esteve, F. (2003). Arquitectura y Documentación: arqueología de la vivienda en el Casco Antiguo de Barcelona. Revista Scripta Nova, 1 de agosto de 2003. España.

- Grout, L. N., Wang, D. (2010). Architectural Research Methods, Second Edition. EEUU. 
- Castillero Calvo, A. (1967) estructuras sociales y económicas de Veragua desde sus orígenes históricos siglo XVI y XVII. Editora Panamá.

- Castillero Calvo, A. (1995) conquista evangelización y resistencia. Editorial Mariano Arosemena.

- Osorio, A (2000). Historia eclesiástica de Panamá: 1815-1915. Grupo Arroba.

- Carles, R.D. (1969) 220 años del período colonial en Panamá. 